\title{
An unusual endoscopic image of a submucosal esophageal hematoma
}

Lei Shi*, and Fu Jian Liu

Department of Gastroenterology, The Eighth Affiliated Hospital of Guangxi Medical University, Guangxi Zhuang Autonomous Region, China

A 44-year-old man was referred to our department with hematemesis after swallowing areca nut for the past 2 days. No underlying diseases were found in this patient. Laboratory results revealed an elevated C-reactive protein level $(13.85 \mathrm{mg} / \mathrm{dl})$. Otherwise his blood coagulates, liver and kidney function were normal. Endoscopy revealed an extensive longitudinal submucosal esophageal hematoma, extending from $20 \mathrm{~cm}$ past the incisors to the gastroesophageal junction. The lumen of the esophagus was moderately congested. No active bleeding or foreign body was seen (Figure 1). After 7 days of fasting and conservative treatment, endoscopic follow-up revealed that the hematoma had completely resolved and a shallow ulcer at the original lesion (Figure 2).

Esophageal hematoma is a very rare phenomenon in clinical practice. Previous reports regarding the causes of esophageal hematoma have referred mainly to complications of endoscopy [1]; other reported cases were caused by hard food boluses and coagulopathy, and trauma, drugs or idiopathic [2,3]. Symptoms of esophageal hematoma are hematemesis, epigastric pain, heartburn, and odynophagia. Differential diagnosis includes aorto-esophageal fistula, esophageal cancer, acute myocardial infarction, esophageal perforation and aortic dissection.

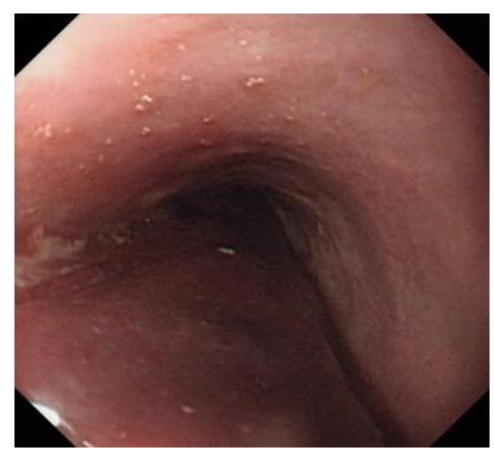

Figure 1. Endoscopy revealed an extensive longitudinal submucosal esophageal hematoma, extending from $20 \mathrm{~cm}$ past the incisors to the gastroesophageal junction.

Copyright: $\mathbb{C} 2016$ Shi L. This is an open-access article distributed under the terms of the Creative Commons Attribution License, which permits unrestricted use, distribution, and reproduction in any medium, provided the original author and source are credited.

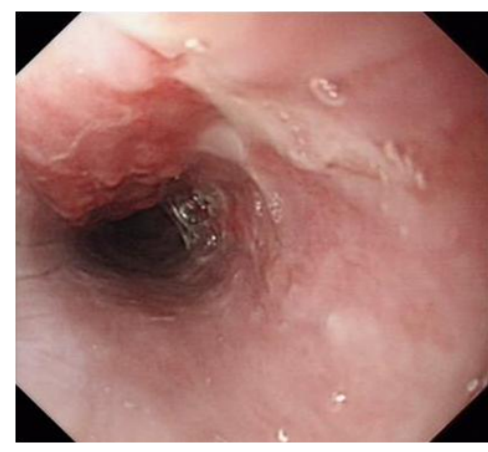

Figure 2. Eendoscopic follow-up revealed that the hematoma had completely resolved and a shallow ulcer at the original lesion.

The management of esophageal intramural hematomas depends on the clinical situation. In most studies, the patients respond well to conservative treatment. However, surgical intervention is required when severe bleeding occurs. We present these findings to raise awareness of the imaging features of this phenomenon. A better understanding of the risk factors involved may also help to avoid misdiagnosis as well as inappropriate treatment of this condition.

\section{References}

1. Tong M, Hung WK, Law S, Wong KH, Kwok KF, Wong J (2006) Esophageal hematoma. Dis Esophagus 19:200-202.

2. Lin AW, Eng M, Robbins D (2009) A very unusual appearance of a rare endoscopic finding: esophageal hematoma. Endoscopy 41 Suppl 2:E40. [Crossref]

3. Chu YY, Sung KF, Ng SC, Cheng HT, Chiu CT (2010) Achalasia combined with esophageal intramural hematoma: case report and literature review. World $J$ Gastroenterol 16:5391-5394. [Crossref]
Correspondence to: Dr. Lei Shi, Department of Gastroenterology The Eighth Affiliated Hospital of Guangxi Medical University, Guangxi Zhuang Autonomous Region China; Fax: +86-775-4200028; E-mail: ycymoment@hotmail.com

Received: October 01, 2016; Accepted: October 25, 2016; Published: October 28,2016 